\title{
ON SOME SEMI-GROUPS
}

\author{
BY REIKICHI YOSHIDA
}

Communicated by N. Jacobson, December 12, 1962

Theorem 1. Let $\Phi$ be an infinite field, $f(x, y)$ a polynomial in $x, y$ over $\Phi$. Let $\alpha, \beta$ be any elements in $\Phi$. In order that $\Phi$ may be a semigroup under the operation $\alpha \circ \beta=f(\alpha, \beta)$, the possible types of $f(x, y)$ are the following:

$$
a x y+b(x+y)+\frac{b(b-1)}{a}, \quad x+y+d, \quad d, \quad x, \quad y,
$$

where $a, b, d$ denote any constants in $\Phi$, and $a \neq 0$.

Moreover under these operations, $\Phi$ is respectively a commutative group with zero, a commutative group, a trivial semi-group (all composites are zero), an "anti-semi-groupe dे droite" and an "anti-semigroupe à gauche."

In the proof of this theorem, after we arrange lexicographically the terms of $f(x, y)$, and compare the leading terms of $(x \circ y) \circ z$ and $x \circ(y \circ z)$, we find that $f(x, y)$ does not include terms of degree $\geqq 3$. Next, from the relations between the coefficients which express that $(x \circ y) \circ z=x \circ(y \circ z)$, we derive five types as a conclusion. Further we obtain Theorem 1 by investigating their structures. Moreover using the result of Theorem 1, we obtain

Theorem 2. Let $\Phi$ be an infinite field, $f(x, y), g(x, y)$ polynomials over $\Phi$. Then, in order that $\Phi$ be an associative semi-ring under the operations $x \oplus y=f(x, y), x \otimes y=g(x, y)$, the possible types are limited to six. Of them, $\Phi$ is a field under

$$
\begin{array}{r}
x \oplus y=x+y+\frac{b^{\prime}}{a^{\prime}}, \quad x \otimes y=a^{\prime} x y+b^{\prime}(x+y)+\frac{b^{\prime}\left(b^{\prime}-1\right)}{a^{\prime}} \\
\quad\left(a^{\prime} \neq 0\right) ;
\end{array}
$$

and $\Phi$ is a trivial ring under

$$
x \oplus y=x+y+d, \quad x \otimes y=-d .
$$

Further, as an application of this theorem, we can see that there exists no lattice with the defining polynomial operations:

$$
x \cup y=f(x, y), \quad x \cap y=g(x, y) .
$$

On the other hand, we lay down the following 
Definition. Let $\bar{S}=\left\{s_{1}, s_{2}, \cdots\right\}$ be a semi-group, and consider the set $S_{i}=\left\{s_{i}, s_{i}^{\prime}, s_{i}^{\prime \prime}, \cdots, s_{i}^{(m)}, \cdots\right\}$, obtained by joining a finite or an infinite number of elements

$$
s_{i}^{\prime}, s_{i}^{\prime \prime}, \cdots, s_{i}^{(m)}, \cdots
$$

to every element $s_{i}$. If we define products in

$$
S=\left\{s_{1}, s_{1}^{\prime}, \cdots, s_{2}, s_{2}^{\prime}, \cdots, \cdots\right\}
$$

as follows:

$$
s_{i}^{(m)} \circ s_{j}^{(n)}=s_{i} \circ s_{j},
$$

$S$ becomes a semi-group, which we shall call the "prolongation" of $\bar{S}$. Then we have

THeOREM 3. Let $\Phi$ be an infinite field and let $a, b, c, d, k ; a^{\prime}, b^{\prime}, c^{\prime}, d^{\prime}, k^{\prime}$ be arbitrary constants in $\Phi$. When we define a new operation by

$(x, y) \circ(z, u)=\left(a x+b y+c z+d u+k, a^{\prime} x+b^{\prime} y+c^{\prime} z+d^{\prime} u+k^{\prime}\right)$,

there exist only 22 types such that $\Phi \times \Phi$ forms a semi-group. They are:

$\left(1^{\circ}\right)$ an anti-semi-groupe à droite: $(x, y) \circ(z, u)=(x, y)$.

$\left(2^{\circ}\right)$ an anti-semi-groupe à gauche: $(x, y) \circ(z, u)=(z, u)$.

$\left(3^{\circ}\right)$ a trivial semi-group: $(x, y) \circ(z, u)=\left(k, k^{\prime}\right)$.

$\left(4^{\circ}\right)$ a commutative group: $(x, y) \circ(z, u)=\left(x+z+k, y+u+k^{\prime}\right)$.

$\left(5^{\circ}\right)$ prolongations of the commutative groups:

$$
\begin{aligned}
&(x, y) \circ(z, u)=\left(x+z+k, \quad a^{\prime}(x+z)+k^{\prime}\right), \\
&(x, y) \circ(z, u)=\left(k, \quad a^{\prime}(x+z)+(y+u)+k^{\prime}\right), \\
&(x, y) \circ(z, u)=(a(x+z)+b(y+u)+k, \\
&\left.\left(\frac{1-a}{b}\right)[a(x+z)+b(y+u)]+k^{\prime}\right) .
\end{aligned}
$$

$\left(^{\circ}\right)$ idempotent semi-groups:

$$
\begin{aligned}
(x, y) \circ(z, u)= & (a(x-z)+b(y-u)+z, \\
& {\left.\left[\frac{a(1-a)}{b}\right](x-z)-a(y-u)+y\right), } \\
(x, y) \circ(z, u)=(x, & \left.a^{\prime}(x-z)+u\right), \\
(x, y) \circ(z, u)=(z, & \left.a^{\prime}(x-z)+y\right) .
\end{aligned}
$$


$\left(7^{\circ}\right)$ prolongations of the anti-semi-groupes a gauche:

$(x, y) \circ(z, u)=\left(z, \quad c^{\prime} z+k^{\prime}\right)$,

$(x, y) \circ(z, u)=\left(k, \quad c^{\prime}(z-k)+u\right)$,

$(x, y) \circ(z, u)=\left(c z+d u+k,\left(\frac{1-c}{d}\right)(c z+d u)-\frac{c k}{d}\right)$.

$\left(8^{\circ}\right)$ prolongations of the anti-semi-groupes a droite:

$(x, y) \circ(z, u)=\left(x, \quad a^{\prime} x+k^{\prime}\right)$,

$(x, y) \circ(z, u)=\left(k, \quad a^{\prime}(x-k)+y\right)$,

$(x, y) \circ(z, u)=\left(a x+b y+k,\left(\frac{1-a}{b}\right)(a x+b y)-\frac{a k}{b}\right)$.

$\left(9^{\circ}\right)$ unions of infinite left ideals:

$$
\begin{aligned}
& (x, y) \circ(z, u)=\left((x+k)+z, \quad a^{\prime}(x+k)+u\right), \\
& (x, y) \circ(z, u)=\left(z, \quad a^{\prime} x+(y+u)+k^{\prime}\right), \\
& (x, y) \circ(z, u)=((a x+b y+k)+z,
\end{aligned}
$$

$$
\left.\left(\frac{1-a}{b}\right)(a x+b y+k)+u\right) .
$$

$\left(10^{\circ}\right)$ unions of infinite right ideals:

$$
\begin{aligned}
(x, y) \circ(z, u) & =\left((z+k)+x, \quad c^{\prime}(z+k)+y\right), \\
(x, y) \circ(z, u) & =\left(x, \quad c^{\prime} z+(y+u)+k^{\prime}\right), \\
(x, y) \circ(z, u) & =((c z+d u+k)+x, \\
& \left.\left(\frac{1-c}{d}\right)(c z+d u+k)+y\right) .
\end{aligned}
$$

$b, d$ denote nonzero constants.

\section{REFERENCES}

1. R. H. Bruck, A survey of binary systems, Springer, Berlin, 1958.

2. E. Hille and R. S. Phillips, Functional analysis and semi-groups, Amer. Math. Soc., Providence, R. I., 1957.

3. N. Jacobson, Lectures in abstract algebra, Van Nostrand, New York, 1951.

4. A. Chatelet et P. Dubreil, Séminaire, demi-groupes, Faculté des Sciences de Paris, Paris, 1956.

Ritsumeikan University, Kyoto, Japan 